\title{
(C) OPEN ACCESS \\ Digital pathology access and usage in the UK: results from a national survey on behalf of the National Cancer Research Institute's CM-Path initiative
}

\author{
Bethany Jill Williams, ${ }^{1}$ Jessica Lee, ${ }^{2}$ Karin A Oien, ${ }^{3}$ Darren Treanor ${ }^{4}$
}

\begin{abstract}
'Histopathology Department St James University Hospital, Leeds, UK

${ }^{2}$ National Cancer Research Institute, London, UK

${ }^{3}$ Institute of Cancer Sciences, College of Medical, Veterinary and Life Sciences, University of Glasgow, Glasgow, UK

${ }^{4}$ Leeds Teaching Hospitals NHS Trust, University of Leeds, Leeds, UK
\end{abstract}

\section{Correspondence to}

Dr Bethany Jill Williams,

Histopathology Department, St

James University Hospital, Leeds LS9 7T, UK; Bethany.williams2@ nhs.net

Received 25 September 2017 Revised 13 November 2017 Accepted 30 November 2017 Published Online First

9 January 2018
Check for updates

To cite: Williams BJ, Lee J, Oien KA, et al. J Clin Pathol 2018:71:463-466.

\section{ABSTRACT}

Aim To canvass the UK pathology community to ascertain current levels of digital pathology usage in clinical and academic histopathology departments, and prevalent attitudes to digital pathology.

Methods A 15-item survey was circulated to National Health Service and academic pathology departments across the UK using the SurveyMonkey online survey tool. Responses were sought at a departmental or institutional level. Where possible, departmental heads were approached and asked to complete the survey, or forward it to the most relevant individual in their department. Data were collected over a 6 -month period from February to July 2017.

Results 41 institutes from across the UK responded to the survey. $60 \%$ (23/39) of institutions had access to a digital pathology scanner, and 60\% (24/40) had access to a digital pathology workstation. The most popular applications of digital pathology in current use were undergraduate and postgraduate teaching, research and quality assurance. Investigating the deployment of digital pathology in their department was identified as a high or highest priority by $58.5 \%$ of institutions, with improvements in efficiency, turnaround times, reporting times and collaboration in their institution anticipated by the respondents. Access to funding for initial hardware, software and staff outlay, pathologist training and guidance from the Royal College of Pathologists were identified as factors that could enable respondent institutions to increase their digital pathology usage. Conclusion Interest in digital pathology adoption in the UK is high, with usage likely to increase in the coming years. In light of this, pathologists are seeking more guidance on safe usage.

\section{INTRODUCTION}

Digital pathology, the examination of digital slides on computer workstations, has long been recognised as an important tool in healthcare education and research. ${ }^{1}$ The evolution of whole slide imaging (WSI) hardware and software over the last decade, and the recent approval of the first WSI device for primary diagnosis in the USA have prompted a surge in interest in clinical digital pathology for primary and secondary diagnoses. ${ }^{2}$ Simultaneously, pathology services are under increasing pressure in terms of workload and work intensity, and it is thought that digital pathology might be part of the solution to emergent staffing issues. ${ }^{3}{ }^{4}$ In light of this, the National Cancer Research Institute's (NCRI) Cellular Molecular Pathology (CM-Path) initiative wanted to canvass the UK pathology community to ascertain current levels of digital pathology usage in clinical and academic histopathology departments.

\section{METHODS}

A survey was developed by members of the technology and informatics workstream of the NCRI's CM-Path initiative. The survey comprised 15 items and assessed: (1) access to and ownership of digital pathology hardware; (2) current and predicted usage of digital pathology; and (3) prevailing attitudes to digital pathology.

The survey was initially circulated to the whole CM-Path membership using the SurveyMonkey online survey tool (www.surveymonkey.com) with specific instructions for completion of forms. As the aim was to assess national trends in digital pathology uptake and attitudes, responses were sought at a departmental or institutional level. Where possible, departmental heads were approached and asked to complete the survey, or forward it to the most relevant individual in their department. Data were collected over a 6-month period from February to July 2017. Reminder emails were sent out during this period, and survey invitations were extended to academic and clinical pathology departments without a CM-Path member. Simple summary statistics were calculated for each questionnaire item. Not all questions were mandatory, and some questions allowed more than one response to be selected per respondent, so denominators are shown for the results on a per question basis.

\section{RESULTS}

A total of 41 questionnaires were completed, representing 41 institutions in England, Wales and Scotland, with no duplications.

\section{Respondent demographics}

Respondent demographics are shown in table 1. The majority of respondents represented National Health Service (NHS) clinical pathology departments $(85 \%, 34 / 40)$, with the remaining 15\% (6/40) of responses from university academic pathology units. Of the 34 clinical pathology departments that responded, 10 were based in district general hospitals, and 24 in tertiary referral centres.

The clinical departments surveyed varied greatly in size, with the number of whole time equivalent consultants ranging from 4 to 47 , and the estimated number of histopathology cases accessioned per year ranging from 2000 to 90000 . 


\begin{tabular}{lll}
\hline Table 1 & Respondent department characteristics & \\
\hline Type of institution & \% of responses & Responses, $\mathbf{n}$ \\
\hline NHS - tertiary referral centre & 60.0 & 24 \\
NHS - district general hospital & 25.0 & 10 \\
University academic department & 15.0 & 6 \\
Total respondents & & 40 \\
\hline
\end{tabular}

NHS, National Health Service.

\section{Access to and ownership of digital pathology hardware}

$60.0 \%$ (23/39) of participating institutions had access to a digital pathology scanner. Of these institutions, 34.8\% (8/23) had an NHS-owned scanner, 43.5\% (10/23) had a university-owned scanner and $21.7 \%(5 / 23)$ had access to a scanner owned by neither the NHS nor the university (see figure 1 ).

$60.0 \%(24 / 40)$ of institutions had access to a digital pathology workstation, but only $46.2 \%$ (18/39) had access to a digital slide archive or library.

\section{Current digital slide usage}

$58.8 \%(20 / 34)$ institutions reported that they do not currently produce any digital slides. Of the institutions that currently produce scanned slides, the annual total ranged from 50 slides to 30000 slides.

The most popular applications of digital pathology in current use were undergraduate and postgraduate teaching, research and quality assurance. Experience with direct clinical use of digital pathology was less widespread, but $31 \%$ of departments indicated they use digital slides for primary diagnosis and $36 \%$ for secondary diagnosis, in a proportion of cases (see figure 2).

\section{Predicted digital slide usage}

When asked to predict their institution's projected usage of digital pathology in 1 year's time, an increased proportion of institutions predicted that digital slides would be used always or often for all digital slide use types (see figure 3 ).

\section{Image analysis usage}

$41.0 \%$ of institutions (16/39) report that they currently use image analysis on digital slides, with immunoscoring, tumour environment assessment, basic measurements, tumour cell proportions and tumour segmentation given as examples of current usage.

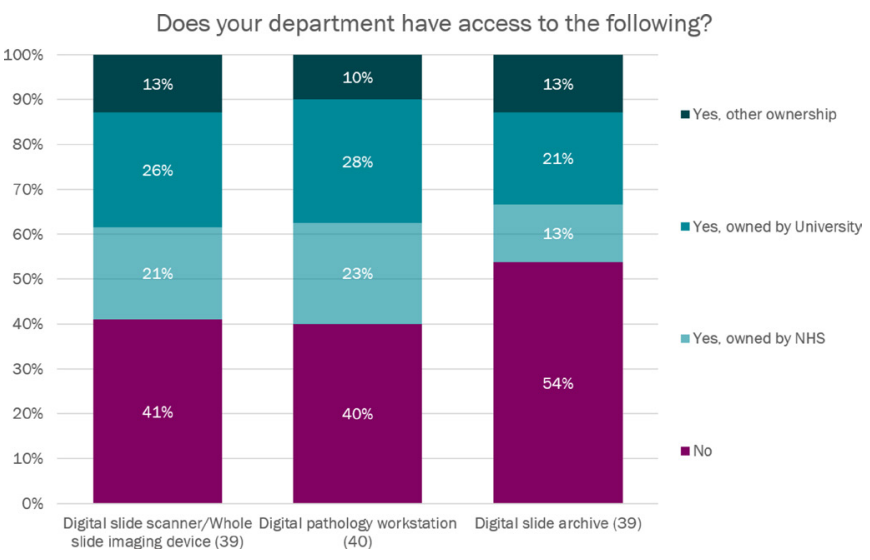

Figure 1 Access to and ownership of digital pathology hardware. NHS, National Health Service.
Today, to what degree does your institution use digital slides for the following scenarios?

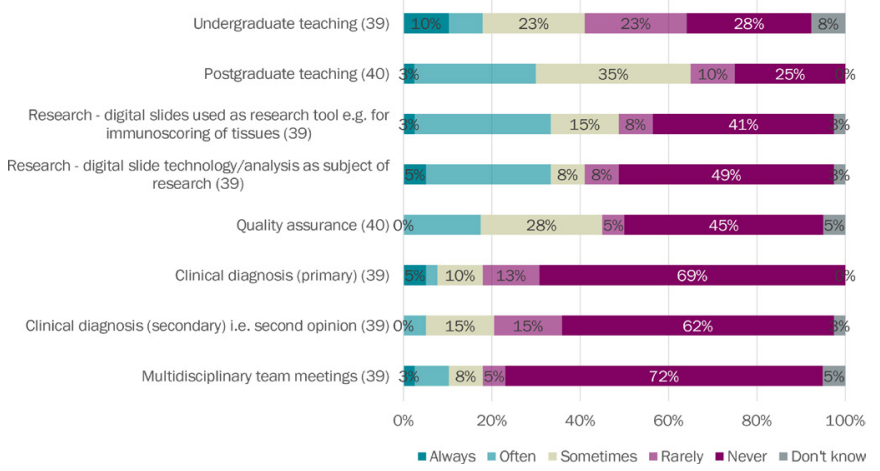

Figure 2 Current usage of digital slides. MDT, multidisciplinary team meeting.

\section{Attitudes to digital pathology adoption and usage}

The majority of departments $(24 / 41,58.5 \%)$ listed the investigation and use of digital pathology as a high or essential priority at their institution (see table 2).

When asked about the perceived benefits of digital pathology for their department, the majority of respondents agreed or strongly agreed that digital pathology would improve efficiency, turnaround times, reporting times and collaboration in their institution (figure 4). Overall laboratory costs and safety were the only parameters that the majority of respondents did not think would be improved by introducing digital pathology.

Respondents were next asked what they perceive to be the barriers to wider digital pathology adoption. $82.5 \%$ of respondents agreed or strongly agreed that initial financial cost was a barrier to wider digital pathology usage at their institution, while only $15 \%$ agreed that safety concerns were impeding more widespread use of digital slides (see figure 5).

Access to funding for initial hardware, software and staff outlay, training for pathologists and guidance from the Royal College of Pathologists were identified as factors that could enable respondent institutions to increase their digital pathology usage (see figure 6).

The following additional enabling factors were identified by respondents, and included as free text:

- Relevant UK data proving cost savings.

- Public Health England (PHE) approval for screening specimens.

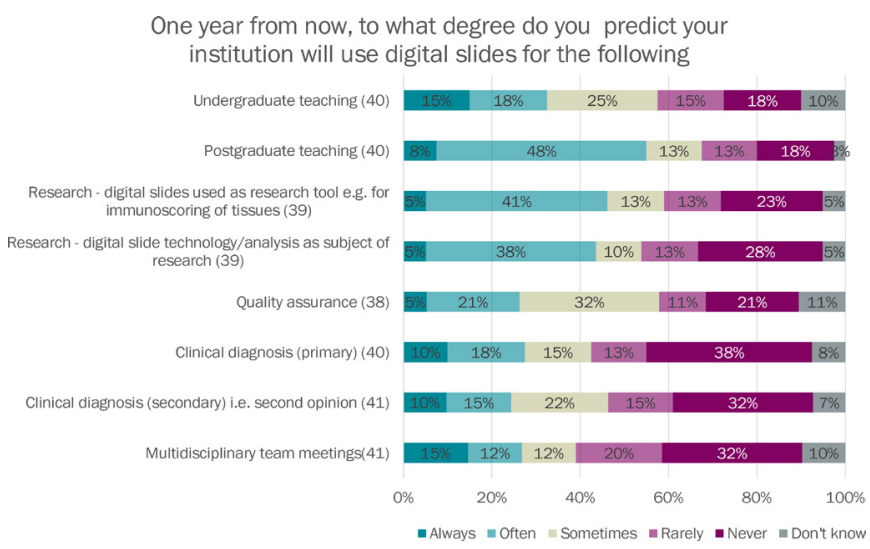

Figure 3 Projected usage of digital slides. MDT, multidisciplinary team meeting. 


\begin{tabular}{lcc}
\hline \multicolumn{2}{l}{ Table 2} & Prioritisation of digital pathology for institutions \\
\hline Digital pathology prioritisation & \% of responses & Responses, $\mathbf{n}$ \\
\hline Not a priority & 9.8 & 4 \\
Low priority & 15 & 6 \\
Neutral & 17 & 7 \\
High priority & 44 & 18 \\
Essential priority & 15 & 6 \\
Total & & 41
\end{tabular}

- NHS England taking a clear and strong stance on digital pathology.

- Improved internet connections.

- Algorithms which improve reporting standards.

- A change in attitude from managers.

- Information technology infrastructure and personnel support.

\section{DISCUSSION}

This NCRI's CM-Path survey was the first attempt to gather national data on access to and usage of digital pathology in NHS and academic pathology departments in the UK. Sixty per cent of respondents had access to WSI scanners, with ownership of these devices split between the NHS and linked university departments. $41.2 \%$ of institutions reported that they currently actively produce digital slides in their department, with the most popular applications being for education, research and quality assurance purposes. Interestingly, $31 \%$ of respondents indicated that they currently use digital slides for primary diagnosis in a proportion of cases, and 36\% use digital slides for secondary diagnosis, indicating that pathology departments are finding utility for the use of digital slides in certain aspects of clinical practice. Predictions for slide usage 1 year from now suggest more departments will be using digital pathology for diagnostic work, and for a greater proportion of cases in the near future.

One of the most interesting findings of the survey was reported level of prioritisation for digital pathology adoption or investigation in the respondent institutions, with the majority of respondents listing it as a high or essential priority. Participants were optimistic that digital pathology could help improve diagnostic efficiency and turnaround time, and 97.6\% agreed or strongly agreed that digital pathology could improve collaboration in their department. Interestingly, patient safety aspects of digital reporting were not emphasised by the survey

In your opinion, what would enable your institution to increase its use of digital pathology?
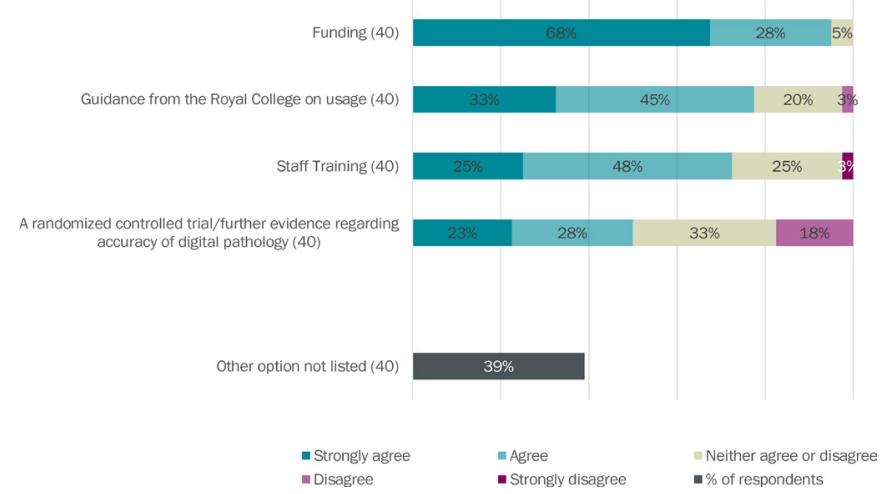

Figure 4 Preferred factors to enable increased use of digital pathology.
What are the current barriers to wider digital pathology usage at your institution?

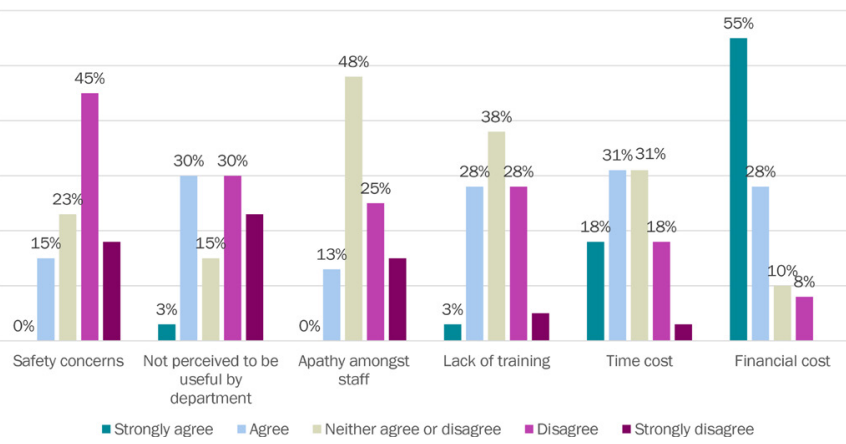

Figure 5 Barriers to digital pathology in your institution.

respondents. Reducing the risk of patient/slide misidentification errors has been identified as one of the key benefits of digital pathology, taking pathology services one step closer to a paperfree workflow. ${ }^{3}$

The most prevalent existing barrier to wider digital adoption for the survey respondents was financial cost to their department. There have been long economic arguments in favour of the introduction of digital pathology (previously known as telepathology) in many contexts ${ }^{3}$ but there is undoubtedly significant initial outlay, in terms of hardware and software, and ongoing training, maintenance and personnel costs. The time required to set up and deploy a system, train staff and ongoing staff time costs to run scanners was also implicated as a barrier for some departments. Clearly, these barriers are counterbalanced by the potential major benefits for diagnostic workflow, workload and workforce issues, and service quality and safety, outlined in a recent review. ${ }^{3}$ Little concern was expressed regarding the safety and accuracy of digital pathology diagnosis versus conventional slide diagnosis, which may reflect the evolving evidence base for digital and glass slide diagnostic concordance. A recent systematic view of ref 5 found a glass:digital concordance rate of $92.4 \%$, and a systematic analysis of glass:digital discordances found that the majority of these discordances were of little or no clinical significance. ${ }^{6}$

$92.5 \%$ of respondents agreed or strongly agreed that funding was required to aid increased uptake of digital pathology in their institution, and $78 \%$ wanted guidance from the Royal College on digital pathology usage. Interestingly, more people identified Royal College guidance as a digital pathology enabler than a randomised controlled trial of digital pathology accuracy. A new Royal College of Pathologist's guideline for digital pathology has been consulted on, and will shortly be published in its final

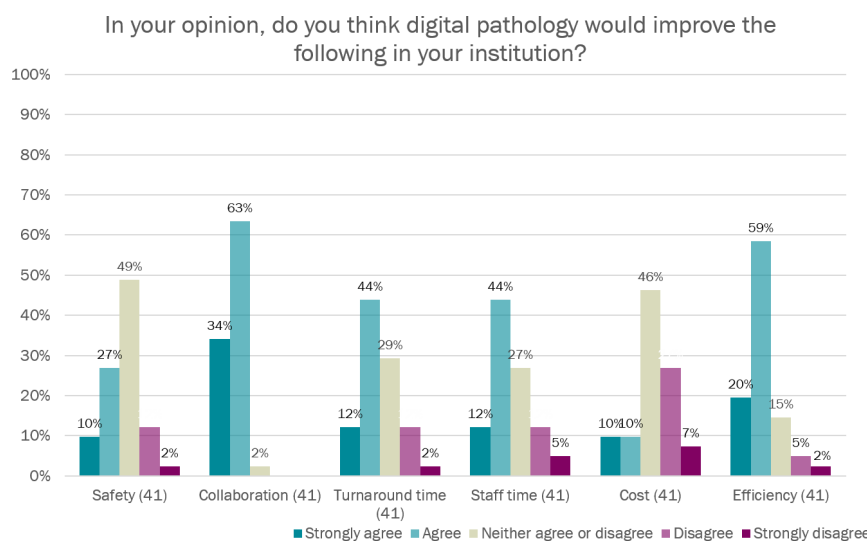

Figure 6 Perceived benefits of digital pathology. 
version, ${ }^{7}$ and may help more departments evaluate the opportunities and risks of digital pathology adoption in their department. In free-text statements, respondents also indicated that a need for direction from NHS England and an approval from PHE were needed to enable them to move forward with digital pathology adoption.

The results of the survey suggest that interest in digital pathology adoption in the UK is high, and that an increasing proportion of pathological diagnosis will be made on digital slides in the immediate future. Furthermore, the recently published Life Sciences Industrial Strategy recognises the need for increased adoption of digital pathology within the NHS, ${ }^{8}$ citing that digital pathology will allow the use of artificial intelligence that could provide prognostic insights that are currently unavailable. To support this adoption, pathology departments would value clear guidelines and statements from key national healthcare, professional and regulatory bodies regarding their position on digital pathology in the clinic, and the necessary steps to take to ensure any adoption maintains or improves on current standards of quality and safety.

\section{Take home messages}

- Access to, and usage of digital pathology hardware is predicted to grow rapidly in the next 12 months.

- Digital pathology adoption has been reported as having a high or essential level of priority for the majority of responding departments.

- Pathology departments are seeking clear guidelines and statements from national healthcare bodies to aid them in safe adoption of digital reporting technology.
Handling editor Dhirendra Govender.

Contributors BJW and DT: study design, drafting and reviewing the manuscript. $\mathrm{JL}$ : data analysis, data collection, reviewing the manuscript. KAO: study design and manuscript review.

Competing interests None declared.

Provenance and peer review Not commissioned; externally peer reviewed.

Open Access This is an Open Access article distributed in accordance with the Creative Commons Attribution Non Commercial (CC BY-NC 4.0) license, which permits others to distribute, remix, adapt, build upon this work non-commercially, and license their derivative works on different terms, provided the original work is properly cited and the use is non-commercial. See: http://creativecommons.org/ licenses/by-nc/4.0/

(C) Article author(s) (or their employer(s) unless otherwise stated in the text of the article) 2018. All rights reserved. No commercial use is permitted unless otherwise expressly granted.

\section{REFERENCES}

1 Cross SS, Dennis T, Start RD. Telepathology: current status and future prospects in diagnostic histopathology. Histopathology 2002;41:91-109.

2 Food and Drug Administration. FDA allows marketing of first whole slide imaging system for digital pathology. Press release 2017. https://www.fda.gov/newsevents/ newsroom/pressannouncements/ucm552742.htm (accessed 8 Apr 2017).

3 Williams BJ, Bottoms D, Treanor D. Future-proofing pathology: the case for clinical adoption of digital pathology. J Clin Pathol 2017;70:1010-8.

4 Cancer Research Uk. Testing times to come? An evaluation of pathology capacity across the UK. http://www.cancerresearchuk.org/sites/default/files/testing_times_to_ come_nov_16_cruk.pdf (accessed 10 Feb 2017).

5 Goacher E, Randell R, Williams B, et al. The diagnostic concordance of whole slide imaging and light microscopy: a systematic review. Arch Pathol Lab Med 2017; 141:151-61.

6 Williams BJ, DaCosta P, Goacher E, et al. A systematic analysis of discordant diagnoses in digital pathology compared with light microscopy. Arch Pathol Lab Med 2017; 141:1712-8.

7 Royal College of Pathologists. Digital pathology guidelines. UK: Royal College Members, 2017.

8 Bell J. Office for life sciences, UK government. Life sciences industrial strategy. https:// www.gov.uk/government/publications/life-sciences-industrial-strategy (accessed 20 Sep 2017). 